\title{
7 Conclusions
}

We have now completed a survey of the existing literature. We have travelled a long way from the simple investment functions of yesteryear, but we are still far from a satisfactory state of affairs. One of the glaring defects in most of the existing work is the neglect of risk and uncertainty. Our ignorance of how entrepreneur's expectations are formed and the naive attempts at including them in our models suggests that more work needs to be done in this field. 